\title{
Phase-dependent spectra in a driven two-level atom
}

\author{
Peng Zhou and S. Swain \\ Department of Applied Mathematics and Theoretical Physics, \\ The Queen's University of Belfast, \\ Belfast BTr 1NN, United Kingdom.
}

()

We propose a method to observe phase-dependent spectra in resonance fluorescence, employing a two-level atom driven by a strong coherent field and a weak, amplitude-fluctuating field. The spectra are similar to those which occur in a squeezed vacuum, but avoid the problem of achieving squeezing over a $4 \pi$ solid angle. The system shows other interesting features, such as pronounced gain without population inversion.

42.50.Hz, 32.80.-t

One of the most striking features of the interaction of a monochromatically driven two-level atom interacting with a squeezed vacuum is the existence of phasedependent features in resonance fluorescence. The relative heights and widths of the spectrum triplet vary greatly with the phase of the squeezed vacuum [1, 2]. In addition, some peaks may show subnatural linewidths. Although these effects were predicted over a decade ago, their experimental verification remains a major challenge in quantum optics. The principal difficulty confronting the experimenter is that the squeezed field modes must occupy the whole $4 \pi$ solid angle of space.

In this paper we propose a method of observing phasedependent resonance fluorescence spectra which avoids this obstacle. The experiment should be feasible with current technology. We employ a two-level atom driven by a strong, coherent laser, and in addition, by a weak, amplitude-fluctuating field of wide bandwidth which replaces the squeezed vacuum. We find similar phasesensitive spectral profiles in resonance fluorescence and probe absorption to those which occur in the squeezed vacuum. A distinction is that no subnatural linewidths arise. Nevertheless, the observation of these features would be an important demonstration of the modification of basic radiative properties by a phase dependent reservoir.

The effect of the relative phase of two coherent driving fields on the transient dynamics of two-level atoms has recently been investigated experimentally [3], the measured fluorescence intensity strongly demonstrating the phase-dependence. Schemes have been proposed using the phase difference of coherent driving fields to control the quantum interference between different transition channels, thereby manipulating spontaneous emission [A] and the shape of the Autler-Townes doublet [5] in multi-level atomic systems. A novel frequency metrological technique and measurements of quantum corre- lations have been demonstrated by means of the phase sensitivity of the rate of two-photon absorption [6]. The relative phase of two strong lasers is also widely employed to control the line shape and rate of multiphoton ionization [7, 8] and the final products of chemical reactions [9].

Very recently, Camparo and Lambropoulos [8] have investigated the effect of phase-diffusion of the fundamental field on 3-photon and 1-photon photoionization, and shown that significant control can be achieved, even in the presence of a large laser linewidth. Measurements of the two-photon absorption spectrum from a variety of randomly amplitude-modulated laser fields, including the real Gaussian field with a coherent component, have been performed [10]. Various spectral profiles are observed when the coherent component is in-phase or 90 degrees out-of-phase with the stochastic component. For the Mollow spectrum, Vemuri et al. 11 have recently shown that the presence of a stochastic field, in addition to a coherent driving field, may give rise to a dramatic narrowing of the linewidths of all three peaks, as well as enhancement of the inversionless gain.

Here we study the modification of the Mollow triplet as controlled by the phase difference between applied coherent and stochastic fields. A single two-level atom is driven by a coherent field with a constant amplitude $E_{c}$, and a stochastic field with a randomly fluctuating amplitude $E_{s}(t)$. The atom is also damped in the usual way by the electromagnetic vacuum. The frequencies of the atomic transition, of the coherent laser and of the stochastic field are assumed to be identical for simplicity. The master equation for the density operator $\rho$ of the system is

$$
\begin{aligned}
\dot{\rho}= & -i\left[H_{a-c}+H_{a-s}, \rho\right] \\
& +\gamma\left(2 \sigma_{-} \rho \sigma_{+}-\sigma_{+} \sigma_{-} \rho-\rho \sigma_{+} \sigma_{-}\right),
\end{aligned}
$$

where

$$
\begin{aligned}
& H_{a-c}=\frac{\Omega}{2}\left(\sigma_{+}+\sigma_{-}\right), \\
& H_{a-s}=\frac{x(t)}{2}\left[e^{i \phi} \sigma_{+}+e^{-i \phi} \sigma_{-}\right] .
\end{aligned}
$$

$H_{a-c}$ and $H_{a-s}$ describe the interaction of the atom with the coherent field and the stochastic field, respectively, $\gamma$ is the atomic decay constant, $\phi$ is the relative phase of the two fields, $\Omega=2\left|\mathbf{d} \cdot \mathbf{e} E_{c}\right| / \hbar$ is the Rabi frequency of the coherent field, and $x(t)=2\left|\mathbf{d} \cdot \mathbf{e} E_{s}(t)\right| / \hbar$ represents the stochastic amplitude of the atom/stochastic-field interaction, which is assumed to be a real Gaussian-Markovian 
random process with zero mean value and correlation function [10,12, 13 ,

$$
\left\langle x(t) x\left(t^{\prime}\right)\right\rangle=D \kappa e^{-\kappa\left|t-t^{\prime}\right|},
$$

where $D$ is the strength of the stochastic process and $\kappa$ can be associated with the bandwidth of the stochastic field. The correlation function (4) describes a field undergoing amplitude fluctuations, which result in a finite laser bandwidth $\kappa$ 10,12,13. The field may be generated by modulating the output of a stabilized tunable ring dye laser external to the laser cavity, and employed to probe the two-photon absorption spectrum [10,12] and resonance fluorescence spectrum [13].

For simplicity, we assume that the intensity of the coherent part is much greater than that of the stochastic field, and the bandwidth $\kappa$ of the stochastic field is much greater than the atomic linewidth (in other words, the correlation time $\kappa^{-1}$ of the stochastic field is very short compared to the radiative lifetime $\gamma^{-1}$ of the atom), i.e.,

$$
\Omega \gg \sqrt{D \kappa} \quad \text { and } \quad \kappa \gg \gamma \text {. }
$$

One can then invoke standard perturbative techniques to eliminate the stochastic variable $x(t)$ [14]. The resultant master equation for the reduced density operator $\rho$ takes the form

$$
\begin{aligned}
\dot{\rho}= & -i\left[H_{a-c}, \rho\right] \\
& +\gamma\left(2 \sigma_{-} \rho \sigma_{+}-\sigma_{+} \sigma_{-} \rho-\rho \sigma_{+} \sigma_{-}\right) \\
& +\left[\left(\sigma_{z} \rho \sigma_{z}-\rho\right),\left(\alpha_{0} \sigma_{+}-\alpha_{0}^{*} \sigma_{-}\right)\right] \\
& +\left[\left(e^{-i \phi} \sigma_{-}+e^{i \phi} \sigma_{+}\right),\left[\rho,\left(\alpha e^{-i \phi} \sigma_{-}+\alpha^{*} e^{i \phi} \sigma_{+}\right)\right]\right]
\end{aligned}
$$

with

$$
\begin{aligned}
& \alpha_{0}=\frac{-i D \Omega \kappa}{8\left(\kappa^{2}+\Omega^{2}\right)}\left(1-e^{i 2 \phi}\right), \\
& \alpha=\frac{D}{8}\left[1+e^{i 2 \phi}+\frac{\kappa^{2}}{\kappa^{2}+\Omega^{2}}\left(1-e^{i 2 \phi}\right)\right] .
\end{aligned}
$$

The first and second terms in the right-hand side of the reduced master equation (6) describe respectively atomic stimulated transitions by the coherent field and spontaneous emission by the vacuum, whereas the other terms are associated with the effect of the stochastic amplitude-fluctuating field, and are phase dependent. It may be shown that the third term acts like a driving field contribution, and the last a reservoirrelated one. In other words, the weak, wide bandwidth and amplitude-fluctuating field effectively forms a phasedependent reservoir.

This becomes clearer if we rewrite the equation (6) for $\phi=0$ as,

$$
\begin{aligned}
\dot{\rho}= & -i\left[H_{a-c}, \rho\right] \\
& +\gamma(N+1)\left(2 \sigma_{-} \rho \sigma_{+}-\sigma_{+} \sigma_{-} \rho-\rho \sigma_{+} \sigma_{-}\right) \\
& +\gamma N\left(2 \sigma_{+} \rho \sigma_{-}-\sigma_{-} \sigma_{+} \rho-\rho \sigma_{-} \sigma_{+}\right) \\
& +2 \gamma M \sigma_{+} \rho \sigma_{+}+2 \gamma M \sigma_{-} \rho \sigma_{-},
\end{aligned}
$$

where $M=N=D / 4 \gamma$. The master equation (8) is the same as that of a coherently driven two-level atom damped by a reservoir in which there is the maximal classical correlation between pairs of photons, a nonideal squeezed vacuum [1] for example. Such a reservoir is sometimes called a "classically squeezed field" (CSF).

We wish to compare this response to that when the stochastic field is replaced by an ideal squeezed vacuum (ISV), where $|M|=\sqrt{N(N+1)}$. The interaction of a two-level atom with the ISV is characterized by the phase $\Phi$, the difference between the phases of the driving laser and the squeezed vacuum [1]. The case $\phi=0$ corresponds to $\Phi=\pi$.

For $\phi=\pi / 2$, we again obtain an equation of the form (8), but this is now identical to the equation for a twolevel atom interacting with a CSF with $\Phi=0$. In this case $N=-M=D / 4 \gamma \times \kappa^{2} /\left(\Omega^{2}+\kappa^{2}\right)$. For $\phi \neq 0, \pi / 2$ equation (8) does not correspond exactly to those for a classically squeezed field.

In Figure 1 we present the resonance fluorescence spectra for the stochastic system with $\Omega=200 \gamma, \kappa=100 \gamma$ and $D=10 \gamma$ in frames (a) and (b) for $\phi=0$ and $\pi / 2$ respectively, where the strong phase dependence is evident. In frames (c) and (d) we give the spectra for the corresponding ideal squeezed vacuum, with $\Omega=20$ and $N=0.25$ in (c), and $N=0.05$ in (d). (For the squeezed vacuum case we have divided the parameters by a factor of ten, in order to obtain experimentally reasonable values for $N$.) The comparison between the spectra for the stochastic system and the system with a squeezed vacuum is striking.

It is the modification of the vacuum reservoir by the weak, amplitude-fluctuating field that strongly affects the physical properties of the atom. For example, the two quadratures, $\sigma_{x}=\sigma_{-}+\sigma_{+}$and $\sigma_{y}=i\left(\sigma_{-}-\sigma_{+}\right)$, of the atomic polarization decay at the different rates

$$
\begin{aligned}
& \gamma_{x}=\gamma+\frac{D \kappa^{2}}{\kappa^{2}+\Omega^{2}} \sin ^{2} \phi, \\
& \gamma_{y}=\gamma+D \cos ^{2} \phi
\end{aligned}
$$

whilst the population inversion $\sigma_{z}$ decays at the rate $\gamma_{z}=\gamma_{x}+\gamma_{y}$. All these decay rates are dependent upon the relative phase and intensities of the driving fields. Clearly, when the coherent field is in-phase with the amplitude-diffusing one, i.e. when $\phi=0$, the decay of the dipole quadrature $\sigma_{x}$ is suppressed $\left(\gamma_{x}=\gamma\right)$, while the other decay rate is enhanced $\left(\gamma_{y}=\gamma+D\right)$. When both the fields are $\pi / 2$ degrees out of phase, however, the situation is reversed. The suppression or enhancement of the polarization decays for certain phases may give rise to rich spectral features.

In Figure 2 we show the global variation of the resonance fluorescence spectra with the phase $\phi$ for the stochastic system with $\Omega=200 \gamma, \kappa=100 \gamma, D=40 \gamma$. The strong phase dependence is clear. When $\phi=0$, the central peak is much narrower and higher than the sidebands. As the phase increases, the centre peak broadens 
and decreases, while the sidebands grow and narrow. For $\phi=\pi / 2$, the central peak has the minimal height and the maximal linewidth, whereas the sidebands have the opposite characteristics. This behaviour is qualitatively similar to that of a two-level atom in a squeezed vacuum [1]. The only essential difference is that there are no subnatural linewidths involved.

The physics associated with the modification of the Mollow fluorescence spectrum can be explored by working in the semiclassical dressed states basis $| \pm\rangle=(|0\rangle \pm$ $|1\rangle) / \sqrt{2}$, which are the eigenstates of $H_{a-c}$. The condition (5) ensures the secular approximation to be valid, and consequently, the equations of motion simplify to

$$
\begin{aligned}
& \dot{\rho}_{z}=-\Gamma_{\|} \rho_{z}, \\
& \dot{\rho}_{+-}=-\left(\Gamma_{\perp}+i \Omega^{\prime}\right) \rho_{+-},
\end{aligned}
$$

where

$$
\begin{aligned}
& \Gamma_{\|}=\gamma_{x}, \quad \Gamma_{\perp}=\frac{1}{2}\left(\gamma_{y}+\gamma_{z}\right), \\
& \Omega^{\prime}=\Omega\left[1+\frac{D \kappa}{2\left(\kappa^{2}+\Omega^{2}\right)} \sin ^{2} \phi\right] .
\end{aligned}
$$

$\rho_{z}=\left(\rho_{++}-\rho_{--}\right)$and $\rho_{+-}=\langle+|\rho|-\rangle$ are the dressedstate inversion and polarization, which have phasedependent decay rates $\Gamma_{\|}$and $\Gamma_{\perp}$, respectively, and $\Omega^{\prime}$ is the generalized Rabi frequency. The quantity $\Omega^{\prime}-\Omega$ represents a dynamical frequency shift owing to the additional amplitude-fluctuating field. (In fact, it is consistent with our approximation (5) to set $\Omega^{\prime}=\Omega$.) Obviously, the impact of the weak stochastic field on the coherently driven atom is merged in the decay rates and level shifts of the dressed states, which depend on the relative phase of the coherent and stochastic fields, the correlation strength $D$ and bandwidth $\kappa$ of the stochastic field.

It follows from $(10)$ that the dressed states $| \pm\rangle$ have the same population. As a result, the resonance fluorescence spectrum is symmetric:

$$
F(\omega)=\frac{\Gamma_{\perp} / 8}{\Gamma_{\perp}^{2}+\left(\omega+\Omega^{\prime}\right)^{2}}+\frac{\Gamma_{\|} / 4}{\Gamma_{\|}^{2}+\omega^{2}}+\frac{\Gamma_{\perp} / 8}{\Gamma_{\perp}^{2}+\left(\omega-\Omega^{\prime}\right)^{2}} .
$$

The decay of the inversion results in the central peak with linewidth $2 \Gamma_{\|}$and height $1 / 4 \Gamma_{\|}$, whereas the modulated decays of the coherences give rise to the sidebands with linewidth $2 \Gamma_{\perp}$ and height $1 / 8 \Gamma_{\perp}$. When $\phi=0$, we have $\Gamma_{\|}=\gamma$ and $\Gamma_{\perp}=3 \gamma / 2+D$. The central spectrum is therefore much narrower and higher the sidebands for $D \gg \gamma$. When $\phi=\pi / 2$, however, $\Gamma_{\|}=$ $\gamma+D \kappa^{2} /\left(\kappa^{2}+\Omega^{2}\right)$ and $\Gamma_{\perp}=3 \gamma / 2+\frac{1}{2} D \kappa^{2} /\left(\kappa^{2}+\Omega^{2}\right)$. Accordingly, the centre peak widens while the sidebands narrow as the phase varies from 0 to $\pi / 2$.

The probe absorption spectrum of such a driven atom is also phase dependent. We show the spectrum in Fig. 3 with the parameters: $\Omega=400 \gamma, \kappa=100 \gamma$ and $D=40 \gamma$. It is clear that the absorption spectra are symmetric only when $\phi=0$ or $\pi / 2$, when the central component exhibits a Lorentzian lineshape, whilst the sidebands show the Rayleigh-wing lineshape. Furthermore, there is a very sharp peak at line centre when $\phi=0$. In these two case, the spectra are qualitatively similar to those of the corresponding ISV [2].

However, for other values of $\phi$ the spectra are asymmetric, and all three resonances have Lorentzian-like lineshapes. A striking feature is the sharp amplification at line centre which occurs in frames (b) and (c). This amplification takes place without the aid of population inversion. (Note that the population can never be inverted in either the bare- or dressed-state basis.) In this respect, the stochastic system differs markedly from the corresponding CSF or ISV, as neither of these systems show strong features at line centre. They display only an insignificant 'glitch' at this position. The stochastic system therefore demonstrate some interesting properties in its own right. A global view of the variation of the probe absorption with phase is presented in Figure 4.

In conclusion, we have reported a scheme to modify the Mollow fluorescence and absorption spectra by means of the relative phase of a coherent field and a stochastically amplitude-diffusing field interacting with a twolevel atom. The phase-sensitive spectral features, which are qualitatively similar to those of a driven atom in a squeezed vacuum, are revealed. In addition, we find some new features of this system, for $\phi \neq 0, \pi$, including inversionless gain. Noting that relevant experiments of the phase-control of the two-photon excitation spectrum of atoms by a field with coherent and real Gaussian components 10], and of the transient dynamics of bichromatically driven two-level atoms [3] have already been demonstrated, the present model is experimentally accessible. It should be possible to observe phase dependent spectra without the necessity to squeeze all $4 \pi$ modes of space. Experiments would also demonstrate our ability to tailor reservoirs so as to modify atomic radiative properties in fundamental ways.

This work is supported by the United Kingdom EPSRC.

[1] H. J. Carmichael et al., Phys. Rev. Lett. 58, 2539 (1987); S. Swain, ibid. 73, 1493 (1994); S. Swain and P. Zhou, Phys. Rev. A 52, 4845 (1995).

[2] H. Ritsch and P. Zoller, Opt. Commun. 64, 523 (1987); S. An et al., ibid. 67, 373 (1988); Z. Ficek et al., Phys. Rev. A 52, 4126(1995); P. Zhou et al., J. Opt. Soc. Am. B 13, 768 (1996).

[3] Q. Wu et al., Phys. Rev. A 49, R1519; 50, 1474 (1994). 
[4] T. Quang et al., Phys. Rev. Lett. 795238 (1997); M. A. G. Martinez et al., Phys. Rev. A 55, 4483 (1997); E. Paspalakis and P. L. Knight, Phys. Rev. Lett. 81, 293 (1998).

[5] U. Lambrecht et al., Phys. Rev. A 57, R3176 (1998).

[6] N. Ph. Georgiades et al., Opt. Lett. 21, 1688 (1996); Phys. Rev. A 55, R1605 (1997).

[7] C. Chen and D. S. Elliott, Phys. Rev. Lett. 65, 1737 (1990); T. Nakajima and P. Lambropoulos, ibid. 70, 1081 (1993); F. Wang et al., ibid. 77, 2416 (1996); M. Protopapas and P. L. Knight, J. Phys. B 28, 4459 (1995); G. X. Li et al., J. Mod. Opt. 44, 505 (1997).

[8] J. C. Camparo and P. Lambropoulos, Phys. Rev. A 55, 552 (1997).

[9] M. Shapiro et al., Chem. Phys. Lett. 149, 451 (1988); P. Brumer, and M. Shapiro, Ann. Rev. Phys. Chem. 43, 257 (1992); L.-C. Zhu et al., Science, 270, 77 (1995); E. Charron et al., Phys. Rev. Lett. 75, 2815 (1995).

[10] C. Chen et al., Phys. Rev. A 49, 461 (1994).

[11] G. Vemuri et al., Phys. Rev. A 41, 2749 (1990); 50, 2599 (1994); P. Zhou and S. Swain, ibid., 58, (in press, 1998).

[12] C. Xie et al., Phys. Rev. A 41, 6376 (1990). G. Klimeck et al., ibid. 44, 3222 (1991); C. Chen et al., Phys. Rev. Lett. 68, 3531 (1992).

[13] A. T. Georges, Phys. Rev. A 21, 2034 (1980); R. E. Ryan and T. H. Bergeman, Phys. Rev. A 43, 6142 (1991).

[14] P. Zhou and S. Swain, Phys. Rev. A 58, 1515 (1998).

FIG. 1. Resonance fluorescence spectrum $F(\omega)$ for $\Omega=200 \gamma, \kappa=100 \gamma, D=10 \gamma$, with (a) $\phi=0$ and (b) $\phi=\pi / 2$. The other frames are for the atom in an ideal squeezed vacuum with $\Omega=20 \gamma$ and $N=0.25, \Phi=\pi$ in (c) and $N=0.05, \Phi=0$ in (d). (All parameters are scaled in units of $\gamma$ throughout these figures.)

FIG. 2. Three-dimensional fluorescence spectrum $F(\omega)$ against the scaled frequency $\omega / \gamma$ and the relative phase $\phi / \pi$, for $\Omega=200 \gamma, \kappa=100 \gamma, D=40 \gamma$.

FIG. 3. Probe absorption spectrum $A(\omega)$, with $\Omega=400 \gamma, \kappa=100 \gamma, D=40 \gamma$, and $\phi=0$ in (a), $\phi=\pi / 6$ in (b), $\phi=\pi / 4$ in (c) and $\phi=\pi / 2$ in (d).

FIG. 4. Same as FIG. 2, but the probe absorption spectrum $A(\omega)$. 
(a)

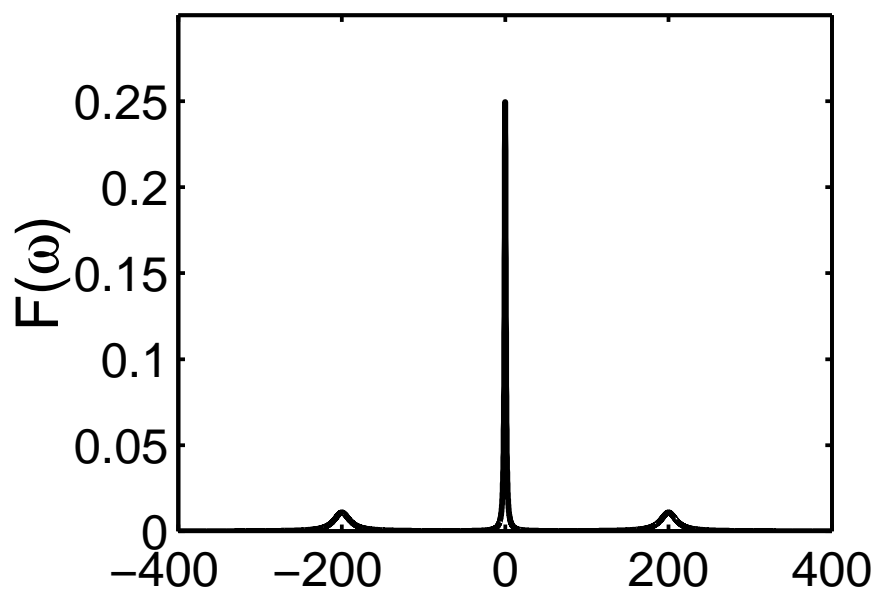

(c)

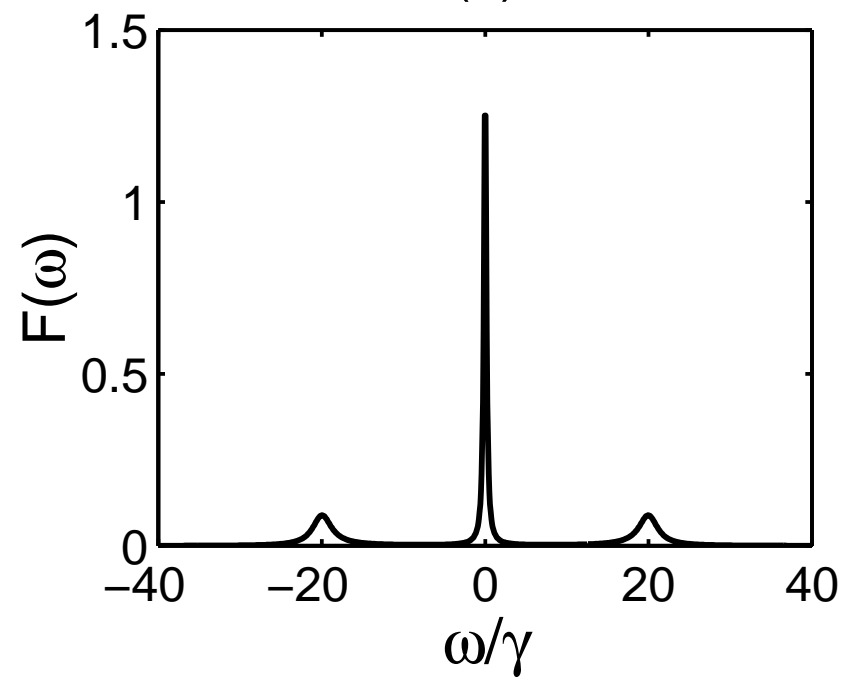

(b)

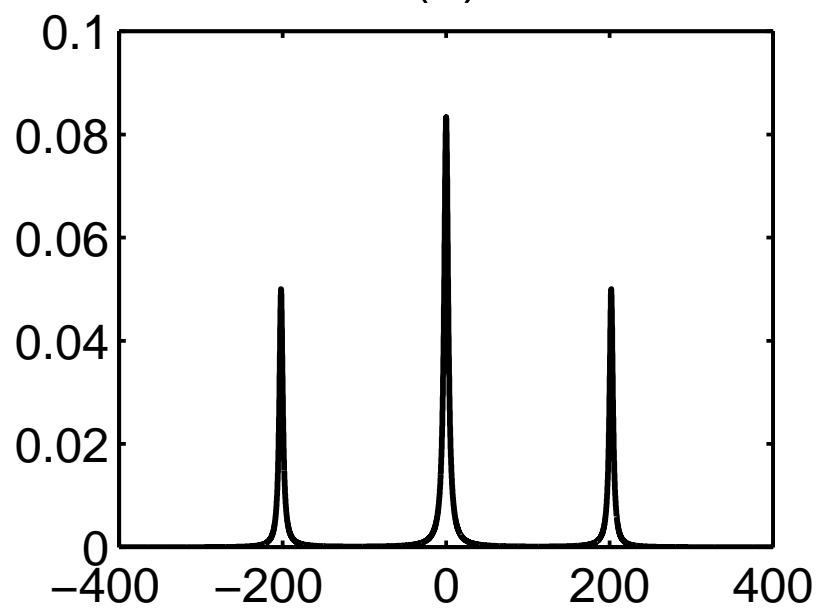

(d)

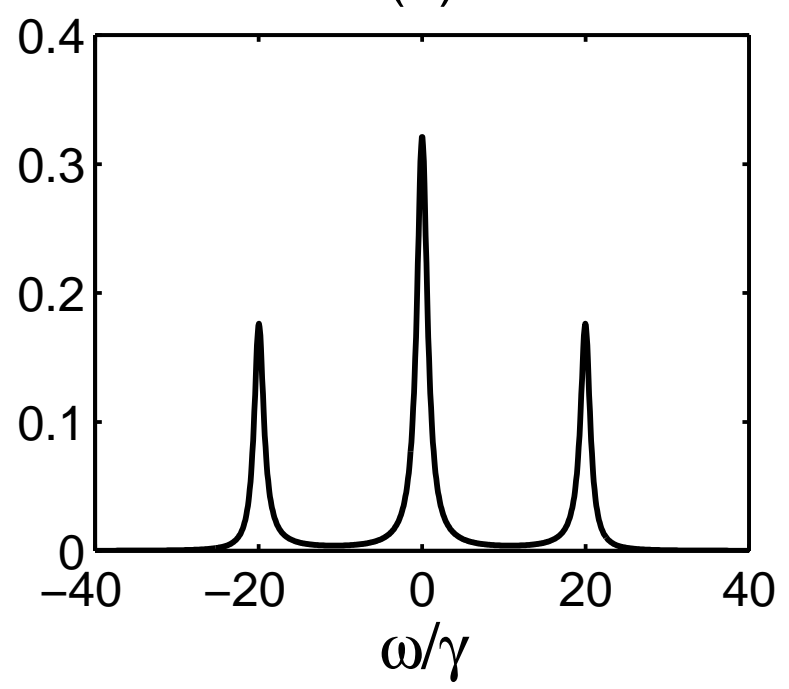




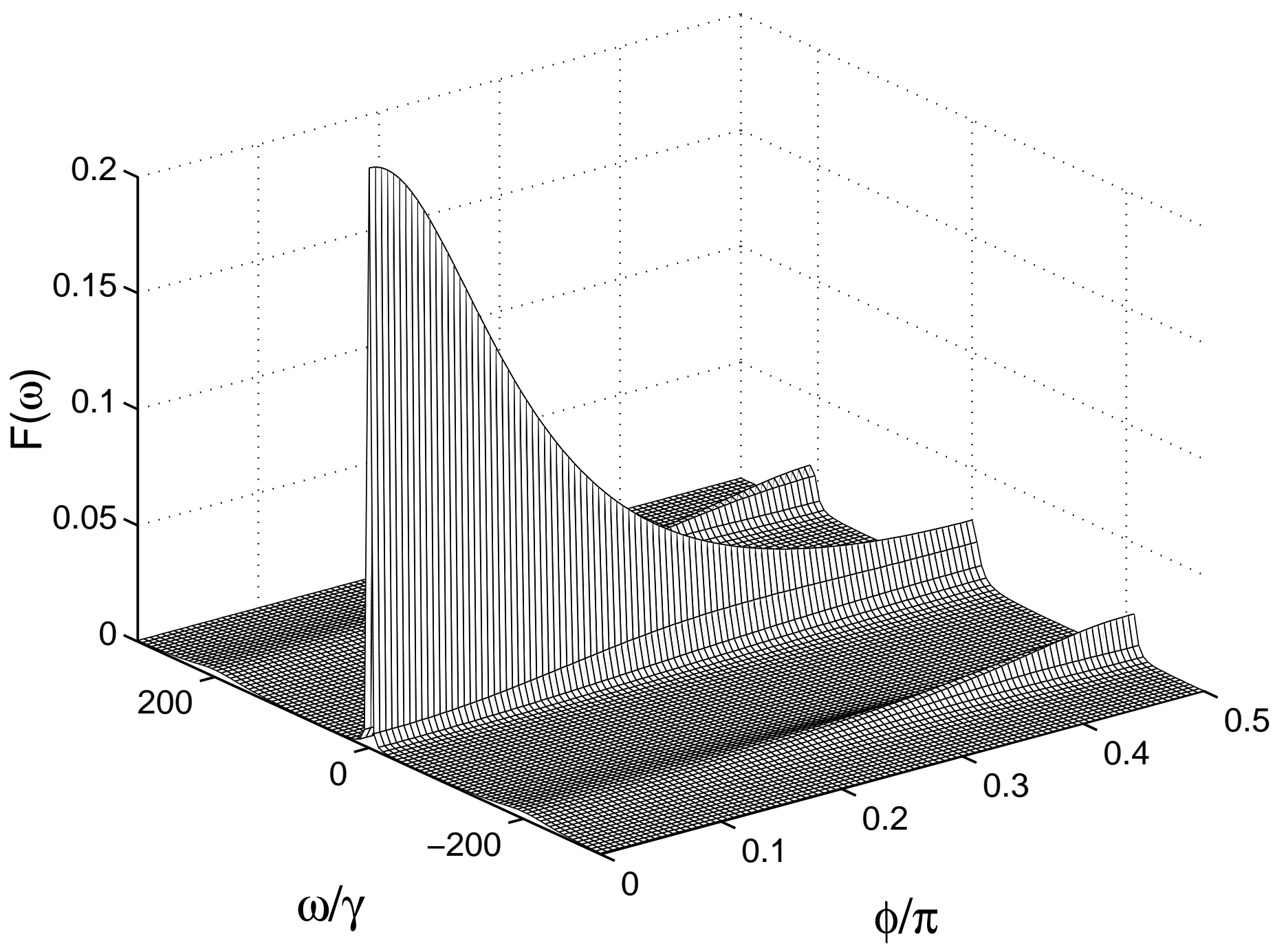



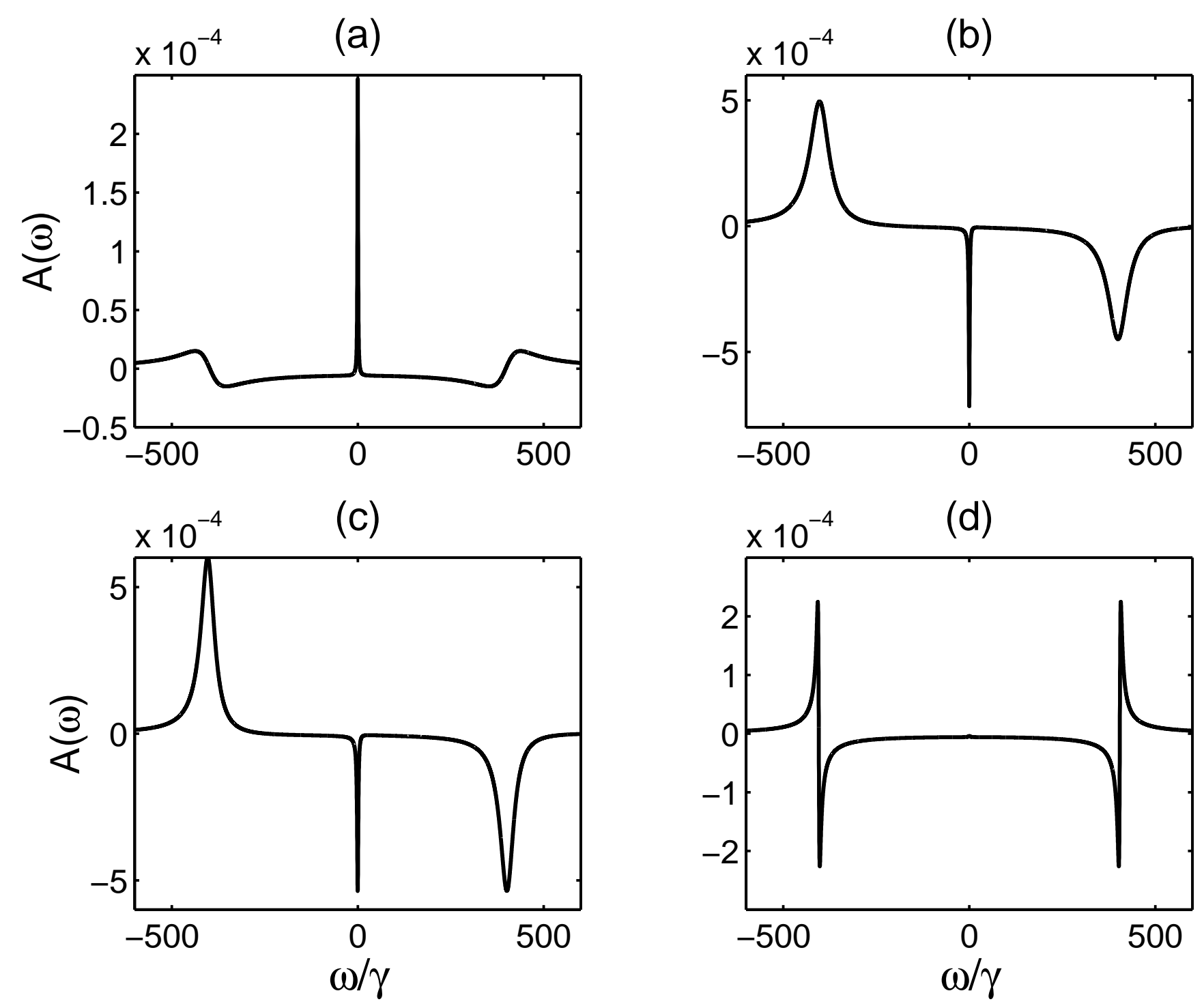


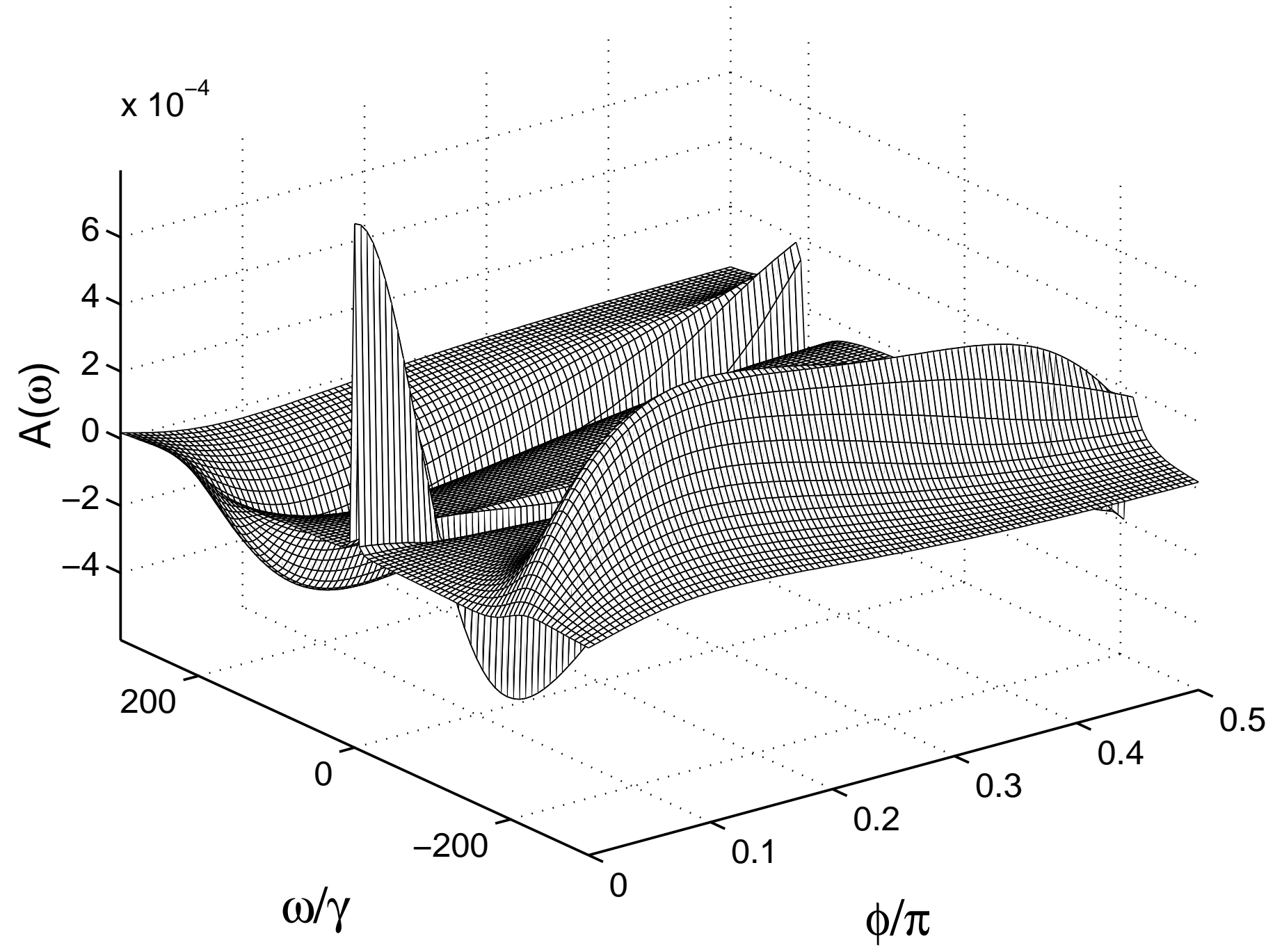

